\begin{tabular}{|r|l|}
\hline \multicolumn{2}{|c|}{ Statistica Sinica Preprint No: SS-2020-0207 } \\
\hline Title & $\begin{array}{l}\text { Nonparametric Maximum Likelihood Estimation Under a } \\
\text { Likelihood Ratio Order }\end{array}$ \\
\hline Manuscript ID & SS-2020-0207 \\
\hline URL & http://www.stat.sinica.edu.tw/statistica/ \\
\hline DOI & $10.5705 /$ ss.202020.0207 \\
\hline Complete List of Authors & $\begin{array}{l}\text { Ted Westling, } \\
\text { Kevin J. Downes and } \\
\text { Dylan Small }\end{array}$ \\
\hline Corresponding Author & Ted Westling \\
\hline E-mail & twestling@math.umass.edu \\
\hline Notice: Accepted version subject to English editing.
\end{tabular}


Statistica Sinica

\title{
NONPARAMETRIC MAXIMUM LIKELIHOOD ESTIMATION UNDER A LIKELIHOOD RATIO ORDER
}

\author{
Ted Westling, Kevin J. Downes, and Dylan S. Small
}

University of Massachusetts Amherst, Children's Hospital of Philadelphia, University of Pennsylvania

Abstract: Comparison of two univariate distributions based on independent sam-

ples from them is a fundamental problem in statistics, with applications in a

variety of scientific disciplines. In many situations, we might hypothesize that

the two distributions are stochastically ordered, meaning that samples from one

distribution tend to be larger than those from the other. One type of stochastic

order is the likelihood ratio order, in which the ratio of the density functions of

the two distributions is monotone non-decreasing. In this article, we derive and

study the nonparametric maximum likelihood estimator of the individual distri-

bution functions and the ratio of their densities under the likelihood ratio order.

Our work applies to discrete distributions, continuous distributions, and mixed

continuous-discrete distributions. We demonstrate convergence in distribution

of the estimator in certain cases, and we illustrate our results using numerical

experiments and an analysis of a biomarker for predicting bacterial infection in

children with systemic inflammatory response syndrome.

Key words and phrases: Biomarker evaluation; Density ratio; Monotonicity constraint; Odds ratio; Ordinal dominance curve; Shape-constrained inference. 


\section{Introduction}

Comparing the distributions of two independent samples is a fundamental problem in statistics. Suppose that $X_{1}, \ldots, X_{n_{1}}$ and $Y_{1}, \ldots, Y_{n_{2}}$ are independent real-valued samples with distribution functions $F_{0}$ and $G_{0}$, respectively. In many situations, we might hypothesize that $F_{0}$ and $G_{0}$ are stochastically ordered, meaning intuitively that samples from $F_{0}$ tend to be larger than those from $G_{0}$. A particular type of stochastic order that arises in many applications is the likelihood ratio order. Specifically, $G_{0}$ and $F_{0}$ satisfy a likelihood ratio order if the density ratio $f_{0} / g_{0}$ is monotone nondecreasing over the support $\mathcal{G}_{0}$ of $G_{0}$, where $f_{0}:=d F_{0} / d \eta$ and $g_{0}:=d G_{0} / d \eta$ for some dominating measure $\eta$. For this reason, the likelihood ratio order is also called a density ratio order.

A likelihood ratio order can arise for a variety of scientific reasons (Beare and Moon, 2015; Roosen and Hennessy, 2004; Dykstra et al., 1995; Yu et al., 2017). In the biomedical sciences and elsewhere, the ratio of two density functions is an object of interest for describing the relative likelihood of a binary status indicator conditional on a covariate. If $D$ is a binary random variable, $Z$ is a scalar random variable, $F_{0}(z)=P(Z \leq z \mid D=1)$, 


$$
\begin{aligned}
G_{0}(z)=P(Z \leq z \mid D=0), \text { and } H_{0}(z):=P(Z \leq z), \text { then } \\
\qquad \frac{f_{0}(z)}{g_{0}(z)}=\frac{\left[d F_{0} / d H_{0}\right](z)}{\left[d G_{0} / d H_{0}\right](z)}=\frac{P(D=1 \mid Z=z) / P(D=1)}{P(D=0 \mid Z=z) / P(D=0)} .
\end{aligned}
$$

Therefore, the density ratio in this context may be interpreted as the relative odds of $D=1$ given $Z=z$ to the overall odds of $D=1$. Since the transformation $x \mapsto x /(1-x)$ is strictly increasing, monotonicity of the density ratio is equivalent to monotonicity of $z \mapsto P(D=1 \mid Z=z)$. One specific situation in which the representation given in $(1.1)$ is of scientific interest is biomarker evaluation, wherein $D$ represents infection status and $Z$ represents the value of a biomarker. Equation (1.1) implies that the ratio of the densities of biomarker values among infected patients to the same among uninfected patients can be interpreted as the odds ratio of infection given biomarker level relative to overall odds of infection. Monotonicity of the density ratio corresponds to the assumption that the conditional probability of infection given biomarker level increases with biomarker level, which is reasonable if the biomarker is actually predictive of disease.

In this article, we derive the nonparametric maximum likelihood estimators of $F_{0}, G_{0}$, and $\theta_{0}=f_{0} / g_{0}$ under the likelihood ratio order restriction and derive certain asymptotic properties of these estimators, including consistency and convergence in distribution. In particular, we use a connection between estimation of $\theta_{0}$ and the classical isotonic regression problem with 
a binary outcome, which both simplifies the derivation of large-sample results and suggests that existing inference methods for the isotonic regression problem can be used to perform inference for $\theta_{0}$ as well. Our results generalize those of Dykstra et al. (1995), who derived the maximum likelihood estimator of $F_{0}$ and $G_{0}$ under a likelihood ratio order in the special case where $F_{0}$ and $G_{0}$ are discrete distributions. We will illustrate our results using numerical experiments and an analysis of a biomarker for predicting bacterial infection in children with systemic inflammatory response syndrome.

Recently, Yu et al. (2017) considered estimation of a monotone density ratio and the individual density functions by maximizing a smoothed likelihood function, and demonstrated certain asymptotic properties of their estimator. Yu et al. (2017) considered maximizing a smoothed likelihood rather than maximizing the likelihood directly because the maximum likelihood estimator of the individual densities does not exist. In contrast, we will show that, using a definition of the likelihood ratio ordered model based on convexity of the ordinal dominance curve, a well-defined nonparametric maximum likelihood estimator of the monotone density ratio function and the individual distribution functions (rather than the density functions) does exist. Furthermore, unlike the smoothed estimator, the derivation of 
the maximum likelihood estimator does not require selection of a bandwidth or any other tuning parameter, and does not rely on the existence of Lebesgue density functions.

Additional relevant references include: Lehmann and Rojo (1992) and Shaked and Shanthikumar (2007), which contain more examples and details regarding stochastic orders, Carolan and Tebbs (2005) and Beare and Moon (2015), which studied tests of the likelihood ratio order, and Rojo and Samaniego (1991), Rojo and Samaniego (1993), Mukerjee (1996), Arcones and Samaniego (2000), Davidov and Herman (2012), and Tang et al. (2017), which considered testing and estimation under other stochastic orders.

\section{Likelihood ratio orders}

We observe two independent real-valued samples $X_{1}, \ldots, X_{n_{1}}$ and $Y_{1}, \ldots$, $Y_{n_{2}}$ with distribution functions $F_{0}$ and $G_{0}$, respectively. We define $\mathcal{F}_{0}$ as the support of $F_{0}$ and $\mathcal{G}_{0}$ as the support of $G_{0}$. We denote $n:=n_{1}+n_{2}$, and by $F_{n}$ and $G_{n}$ the empirical distribution functions of $X_{1}, \ldots, X_{n_{1}}$ and $Y_{1}, \ldots, Y_{n_{2}}$, respectively. We define $x_{1}<\cdots<x_{m_{1}}$ as the unique values of $X_{1}, \ldots, X_{n_{1}}, y_{1}<\cdots<y_{m_{2}}$ as the unique values of $Y_{1}, \ldots, Y_{n_{2}}$, and $z_{1}<z_{2}<\cdots<z_{m}$ as the unique values of $\left(X_{1}, \ldots, X_{n_{1}}, Y_{1}, \ldots, Y_{n_{2}}\right)$. Throughout, we assume that $n$ is fixed, but that $n_{1}$ is drawn from a 
$\operatorname{Binomial}\left(n, \pi_{0}\right)$ distribution for some $\pi_{0} \in(0,1)$.

We let $\mathcal{D}$ be the space of distribution functions on $\mathbb{R}$; i.e. all nondecreasing, cádlàg functions $H$ such that $\lim _{x \rightarrow-\infty} H(x)=0$ and $\lim _{x \rightarrow \infty} H(x)=$ 1. For any nondecreasing function $h: \mathbb{R} \rightarrow \mathbb{R}$, we define its generalizedinverse $h^{-}$pointwise as $h^{-}(u):=\inf \{x: h(x) \geq u\}$. When $h \in \mathcal{D}, h^{-}$is called the quantile function of $h$. For any interval $I \subseteq \mathbb{R}$ and any function $h: I \rightarrow \mathbb{R}$, we define the greatest convex minorant (GCM) of $h$ on $I$, denoted $\mathrm{GCM}_{I}(h): I \rightarrow \overline{\mathbb{R}}$, for $\overline{\mathbb{R}}$ the extended real line, as the pointwise supremum of all convex functions on $I$ bounded above by $h$. The least concave majorant operator is defined analogously. We say a function $H$ is convex over a set $\mathcal{S} \subseteq \mathbb{R}$ if for every $x, y \in \mathcal{S}$ and $\lambda \in[0,1]$ such that $\lambda x+(1-\lambda) y \in \mathcal{S}, H(\lambda x+(1-\lambda) y) \leq \lambda H(x)+(1-\lambda) H(y)$. We also define $\partial_{-}$as the left derivative operator for a left differentiable function and $\operatorname{Im}(h):=\{h(x): x \in \mathcal{S}\}$ as the image of a function $h$ defined on a domain $\mathcal{S}$.

The unrestricted nonparametric model for the pair $(F, G)$ of distribution functions of the observed data is $\mathcal{M}_{N P}:=\mathcal{D}^{2}$. As mentioned in the introduction, the likelihood ratio order can be defined as the ratio of the density functions $f_{0}$ and $g_{0}$ of $F_{0}$ and $G_{0}$ with respect to some dominating measure $\eta$ being non-decreasing. By varying the dominating measure, both 
discrete and continuous distributions can be handled this way. However, as noted by Yu et al. (2017), this definition does not lend itself to the derivation of a maximum likelihood estimator, since the likelihood defined through the densities can be made arbitrarily large. Instead, other authors have defined the likelihood ratio order as convexity of the ordinal dominance curve, defined as $t \mapsto R_{F, G}(t):=F \circ G^{-}(t)$ for $t \in[0,1]$ (Bamber, 1975; Hsieh and Turnbull, 1996). Lehmann and Rojo (1992) demonstrated the equivalence of this definition to that using the density functions in the special case that $F$ and $G$ are strictly increasing and continuous on their supports, which were assumed to be intervals. Alternatively, Shaked and Shanthikumar (2007) defined the likelihood ratio order as $F(A) G(B) \leq F(B) G(A)$ for all measurable sets $A, B \subseteq \mathbb{R}$ with $A \leq B$, where $F(A):=\int_{A} d F$ (with some abuse of notation) and $A \leq B$ means that $a \leq b$ for all $a \in A$ and $b \in B$.

In Theorem 1 below, we consolidate and generalize existing results connecting these different definitions of the likelihood ratio order.

Theorem 1. If $F \ll G$ and $\nu:=d F / d G$ is continuous on the support $\mathcal{G}$ of $G$, then (1) the following are equivalent: $R_{F, G}$ is convex on $\operatorname{Im}(G), \nu$ is nondecreasing on $\mathcal{G}$, and $F(A) G(B) \leq F(B) G(A)$ for all measurable sets $A \leq$ $B$; and (2) if $\nu$ is non-decreasing on $\mathcal{G}$ then $\nu(x)=\partial_{-} \operatorname{GCM}_{[0,1]}\left(R_{F, G}\right) \circ G(x)$ for all $x \in \mathcal{G}$. 
To our knowledge, Theorem 1 is the most general result to-date connecting the three definitions of the likelihood ratio ordered model. We note that the three definitions may not be equivalent when $F$ is not dominated by $G$ or $\nu$ is not continuous. For instance, in the proof of Theorem 1 part (1), we only use the assumption that $\nu$ is continuous on $\mathcal{G}$ to show that $R_{F, G}$ is convex on $\operatorname{Im}(G)$ implies that $\nu$ is non-decreasing, but we show that if $\nu$ is non-decreasing, then $F(A) G(B) \leq F(B) G(A)$ for all $A \leq B$ (the definition used in Shaked and Shanthikumar, 2007) regardless of whether $\nu$ is continuous. Additionally, we show that $F(A) G(B) \leq F(B) G(A)$ for all $A=\left(a_{1}, b_{1}\right] \leq B=\left(b_{1}, b_{2}\right]$ implies that $R_{F, G}$ is convex on $\operatorname{Im}(G)$ regardless of whether $F \ll G$ or $\nu$ is continuous. However, to show the converse, we use $F \ll G$. For a simple counterexample when $F$ is not dominated by $G$, consider $F(\{a\})=1$ and $G(\{b\})=1$, where $a<b$. Then $R_{F, G}(u)=I(u>0)$ for $u \in[0,1]$, which is convex on $\operatorname{Im}(G)=\{0,1\}$, but $1=F(A) G(B)>F(B) G(A)=0$ for $A=\{a\} \leq\{b\}=B$. Finally, when $F \ll G$ but $d F / d G$ is not continuous on $\mathcal{G}$, whether $R_{F, G}$ being convex on $\operatorname{Im}(G)$ implies that $F(A) G(B) \leq F(B) G(A)$ for all measurable sets $A \leq B$, or even all such Borel sets, is unclear to us.

Throughout the remainder of the article, we say $(F, G) \in \mathcal{M}_{N P}$ satisfy a likelihood ratio order, and write $G \leq_{L R} F$ if $R_{F, G}$ is convex on $\operatorname{Im}(G)$. 
We then define the likelihood ratio ordered model $\mathcal{M}_{L R}$ as all $(F, G) \in$ $\mathcal{M}_{N P}$ such that $G \leq_{L R} F$. For any $(F, G) \in \mathcal{M}_{N P}$, we further define $\theta: \mathcal{M}_{N P} \rightarrow \Theta$ as $\theta_{F, G}:=\partial_{-} \operatorname{GCM}_{[0,1]}\left(R_{F, G}\right) \circ G$, where $\Theta$ is defined as the set of non-negative, non-decreasing functions on $\mathbb{R}$. We note that this definition allows for the possibility that $F$ is not dominated by $G$, but by Theorem 1, for all $(F, G) \in \mathcal{M}_{L R}$ such that $F \ll G$ and $d F / d G$ is continuous on $\mathcal{G}, \theta_{F, G}=d F / d G$ on $\mathcal{G}$. We define $\theta_{0}:=\theta_{F_{0}, G_{0}}$.

In the context of the likelihood ratio order, many existing works either assume that $F_{0}$ and $G_{0}$ are discrete (e.g. Dykstra et al., 1995) or that $F_{0}$ and $G_{0}$ are continuous (e.g. Lehmann and Rojo, 1992; Yu et al., 2017). In the discrete setting, if $F_{0}$ and $G_{0}$ are discrete distributions with common support and mass functions $\Delta F_{0}$ and $\Delta G_{0}$ such that $\left(F_{0}, G_{0}\right) \in \mathcal{M}_{L R}$, then $\theta_{0}=\Delta F_{0} / \Delta G_{0}$ on $\mathcal{G}_{0}$. Alternatively, if $F_{0}$ and $G_{0}$ both possess Lebesgue density functions $f_{0}$ and $g_{0}$ and $\left(F_{0}, G_{0}\right) \in \mathcal{M}_{L R}$, then $\theta_{0}=f_{0} / g_{0}$ on $\mathcal{G}_{0}$. However, for the purpose of deriving a maximum likelihood estimator, we will demonstrate that these two cases do not need to be treated separately. Furthermore, in some applied settings, $F_{0}$ and $G_{0}$ are neither discrete nor continuous, but rather a mixture of discrete and continuous components, and we will derive results that apply in these situations as well. For instance, exposures that are bounded below may have positive mass at their lower 
boundary, and be continuous thereafter. Many biomarkers exhibit this property. Similarly, some measurements are "clumpy", exhibiting positive mass at integers or other "round" numbers due to the measurement process, but also possessing positive Lebesgue density between such points. In all cases, $\theta_{0}$ has a meaningful interpretation as the ratio of the conditional odds of a sample being from the distribution $F_{0}$ to the unconditional odds of a sample being from $F_{0}$.

\section{Estimation under a likelihood ratio order}

\subsection{Maximum likelihood estimator}

The pair $\left(F_{0}, G_{0}\right)$ determines the joint distribution of the observed data. Defining the nonparametric likelihood of the observed data as $L_{n}(F, G):=$ $\prod_{i=1}^{n_{1}}\left[F\left(X_{i}\right)-F\left(X_{i}-\right)\right] \prod_{j=1}^{n_{2}}\left[G\left(Y_{j}\right)-G\left(Y_{j}-\right)\right]$, the nonparametric maximum likelihood estimator of $\left(F_{0}, G_{0}\right)$, i.e. in the model $\mathcal{M}_{N P}$, is $\left(F_{n}, G_{n}\right)$ for $F_{n}$ the empirical distribution function of $X_{1}, \ldots, X_{n_{1}}$, and $G_{n}$ the same of $Y_{1}, \ldots, Y_{n_{2}}$. This suggests taking as an estimator of $\theta_{0}$ the plug-in estimator $\theta_{n}:=\theta_{F_{n}, G_{n}}=\partial_{-} \operatorname{GCM}_{[0,1]}\left(F_{n} \circ G_{n}^{-}\right) \circ G_{n}$. The function $F_{n} \circ G_{n}^{-}$is known as the empirical ordinal dominance curve, and is properties were studied by Hsieh and Turnbull (1996).

In this section, we demonstrate, amongst other results, that $\theta_{n}$ is the 
3.1 Maximum likelihood estimator11

maximum likelihood estimator of $\theta_{0}$ in the likelihood ratio ordered model $\mathcal{M}_{L R}$. A maximum likelihood estimator of $\left(F_{0}, G_{0}\right)$ in $\mathcal{M}_{L R}$ is defined as $\left(F_{n}^{*}, G_{n}^{*}\right) \in \operatorname{argmax}_{(F, G) \in \mathcal{M}_{L R}} L_{n}(F, G)$, and a maximum likelihood estimator of $\theta_{0}$ is defined as $\theta_{n}^{*}:=\theta_{F_{n}^{*}, G_{n}^{*}}$.

We define $H_{n}(z):=\pi_{n} F_{n}(z)+\left(1-\pi_{n}\right) G_{n}(z)$ as the empirical distribution of the combined sample $X_{1}, \ldots, X_{n_{1}}, Y_{1}, \ldots, Y_{n_{2}}$, and $h_{k}:=H_{n}\left(y_{k}\right)$ for $k=$ $1, \ldots, m_{2}$. Our first result characterizes $\left(F_{n}^{*}, G_{n}^{*}\right)$.

Theorem 2. Let $A_{k}^{*}$ be the value at $h_{k}$ of the $G C M$ over $\left[0, h_{m_{2}}\right]$ of $\left\{\left(h_{k}, F_{n}\left(y_{k}\right)\right)\right.$ : $\left.k=0, \ldots, m_{2}\right\}$ and $B_{k}^{*}$ be the value at $h_{k}$ of the $L C M$ over $\left[0, h_{m_{2}}\right]$ of $\left\{\left(h_{k}, G_{n}\left(y_{k}\right)\right): k=0, \ldots, m_{2}\right\}$. Then $G_{n}^{*}$ is a right-continuous step function with jumps at $y_{1}, \ldots, y_{m_{2}}$ with $G_{n}^{*}\left(y_{k}\right)=B_{k}^{*}$ and $F_{n}^{*}$ is given by a rightcontinuous step function with jumps at $z_{1}, \ldots, z_{m}$, where $F_{n}^{*}\left(y_{k}\right)=A_{k}^{*}$, and for any $x_{i}$ such that $y_{j-1}<x_{i} \leq y_{j}$, where $y_{0}:=-\infty$, the mass of $F_{n}^{*}$ at $x_{i}$ is given by

$$
F_{n}^{*}\left(x_{i}\right)-F_{n}^{*}\left(x_{i}-\right)=\left[F_{n}^{*}\left(y_{j}\right)-F_{n}^{*}\left(y_{j-1}\right)\right] \frac{F_{n}\left(x_{i}\right)-F_{n}\left(x_{i}-\right)}{F_{n}\left(y_{j}\right)-F_{n}\left(y_{j-1}\right)} .
$$

For any $x_{i}$ such that $y_{m_{2}}<x_{i}$, the mass of $F_{n}^{*}$ at $x_{i}$ is given by

$$
F_{n}^{*}\left(x_{i}\right)-F_{n}^{*}\left(x_{i}-\right)=\left[1-F_{n}^{*}\left(y_{m_{2}}\right)\right] \frac{F_{n}\left(x_{i}\right)-F_{n}\left(x_{i}-\right)}{1-F_{n}\left(y_{m_{2}}\right)} .
$$

We also note that $F_{n}^{*}\left(y_{k}\right)=\operatorname{GCM}_{\left[0, h_{m_{2}}\right]}\left(F_{n} \circ H_{n}^{-}\right)\left(H_{n}\left(y_{k}\right)\right)$ and $G_{n}^{*}\left(y_{k}\right)=$ $\operatorname{LCM}_{\left[0, h_{m_{2}}\right]}\left(G_{n} \circ H_{n}^{-}\right)\left(H_{n}\left(y_{k}\right)\right)$. 
3.1 Maximum likelihood estimator12

A proof of Theorem 2, and proofs of all other theorems, are provided in Supplementary Material. We note that $F_{n}^{*}$ necessarily has jumps at all $x_{i}$ and at all $y_{j}$ such that $y_{j} \geq x_{1}$, and $G_{n}^{*}$ has jumps at all $y_{j}$. We also note that if there are $j$ such that no $x_{i} \in\left(y_{j}, y_{j+1}\right]$ but $F_{n}^{*}\left(y_{j}\right)>F_{n}^{*}\left(y_{j-1}\right)$, then there are infinitely many maximizers $F_{n}^{*}$ because any $F_{n}^{*}$ that assigns mass $F_{n}^{*}\left(y_{j}\right)-F_{n}^{*}\left(y_{j-1}\right)$ to the interval $\left(y_{j}, y_{j+1}\right]$ yields the same likelihood and satisfies the constraints. In these cases, for the sake of uniqueness, we will put $\operatorname{mass} F_{n}^{*}\left(y_{j}\right)-F_{n}^{*}\left(y_{j-1}\right)$ at the point $y_{j+1}$.

Theorem 2 implies the following result characterizing $\theta_{n}^{*}$.

Corollary 1. The points $\left\{\left(G_{n}^{*}\left(y_{k}\right), F_{n}^{*}\left(y_{k}\right)\right): k=1, \ldots, m_{2}\right\}$ lie on the GCM over $[0,1]$ of the empirical ordinal dominance curve

$$
\left\{\left(G_{n}\left(y_{j}\right), F_{n}\left(y_{j}\right)\right): k=0, \ldots, m_{2}\right\}
$$

where $y_{0}:=-\infty$. Specifically, if $\left\{\left(h_{j_{k}}, F_{n}\left(y_{j_{k}}\right)\right): k=0, \ldots, K\right\}$ are the vertices of the $G C M$ of $\left\{\left(h_{k}, F_{n}\left(y_{k}\right)\right): k=0, \ldots, m_{2}\right\}$, then $\left(G_{n}\left(y_{j_{k}}\right), F_{n}\left(y_{j_{k}}\right)\right)$ : $k=0, \ldots, K\}$ are the vertices of the GCM of the empirical ordinal dominance curve. Therefore, $\theta_{n}^{*}:=\theta_{F_{n}^{*}, G_{n}^{*}}$ is equal to $\theta_{n}:=\theta_{F_{n}, G_{n}}$.

Theorem 2 bears resemblance to, but is different than, Theorem 2.1 of Dykstra et al. (1995), which characterized the maximum likelihood estimator under a likelihood ratio order in the discrete case. Here, we perform 
3.2 Representation as a transformation of isotonic regression13

the maximization over all pairs of univariate distribution functions $(F, G)$ such that $R_{F, G}=F \circ G^{-}$is convex on the support of $G$, whereas Theorem 2.1 of Dykstra et al. (1995) performed the maximization over $(F, G)$ with support contained in $\left\{z_{1}, \ldots, z_{m}\right\}$ and such that $\left[\Delta F\left(z_{j}\right)\right] /\left[\Delta G\left(z_{j}\right)\right]$ is nondecreasing. The first set is strictly larger than the second, which results in possibly different maximum likelihood estimators. In particular, our maximum likelihood estimator $G_{n}^{*}$ is only supported on $y_{1}, \ldots, y_{m_{2}}$, whereas the maximum likelihood estimator of $G_{0}$ derived by Dykstra et al. (1995) may have support on $x_{j}$ that are not equal to any $y_{1}, \ldots, y_{m_{2}}$. This difference makes sense in the context of our respective problem formulations: Dykstra et al. (1995) assumed that the supports of $F_{0}$ and $G_{0}$ are subsets of $\left\{z_{1}, \ldots, z_{m}\right\}$, while we do not assume the supports are known $a$ priori. In Supplementary Material, we illustrate the use of Theorem 2 using hypothetical data in which our maximum likelihood estimators $F_{n}^{*}$ and $G_{n}^{*}$ are different from those of Dykstra et al. (1995).

\subsection{Representation as a transformation of isotonic regression}

Dykstra et al. (1995) and Carolan and Tebbs (2005) provided representations of the maximum likelihood estimators of $F_{0}$ and $G_{0}$ in terms of isotonic regression in the discrete and continuous cases, respectively. Here, we show 
3.2 Representation as a transformation of isotonic regression14

that $\theta_{n}^{*}$ can also be represented as a transformation of an isotonic regression, which aids in deriving its asymptotic properties. We let $D_{1}, \ldots, D_{n}$ be independent Bernoulli random variables with common probability $\pi_{0}$ and such that $n_{1}=\sum_{i=1}^{n} D_{i}$. Letting $j_{1}, \ldots, j_{n_{1}}$ be the indices such that $D_{j_{i}}=1$ for each $i$, we then define $Z_{j_{i}}:=X_{i}$ for each $i=1, \ldots, n_{1}$. Similarly, letting $k_{1}, \ldots, k_{n_{2}}$ be the indices such that $D_{k_{i}}=0$ for each $i$, we define $Z_{k_{i}}:=Y_{i}$ for each $i=1, \ldots, n_{2}$. Defining the data unit $\mathbf{O}_{i}:=\left(Z_{i}, D_{i}\right)$, observing the independent samples $X_{1}, \ldots, X_{n_{1}}$ from $F_{0}$ and $Y_{1}, \ldots, Y_{n_{2}}$ from $G_{0}$ is then equivalent to observing independent observations $\mathbf{O}_{1}, \ldots, \mathbf{O}_{n}$ from $P_{0}$, where $P_{0}$ satisfies

$$
P_{0}(Z \leq z, D=d)=d \pi_{0} F_{0}(z)+(1-d)\left(1-\pi_{0}\right) G_{0}(z)
$$

Thus, $Z_{1}, \ldots, Z_{n}$ represent the pooled values of $X_{1}, \ldots, X_{n_{1}}, Y_{1}, \ldots, Y_{n_{2}}$, and each $D_{i}$ represents an indicator that $Z_{i}$ corresponds to a sample from $F_{0}$. Furthermore, $F_{0}(z)=P_{0}(Z \leq z \mid D=1), G_{0}(z)=P_{0}(Z \leq z \mid D=0)$, and $\pi_{0}:=P_{0}(D=1)$. Estimating $\theta_{0}$ given the independent samples $X_{1}, \ldots, X_{n_{1}}$ and $Y_{1}, \ldots, Y_{n_{2}}$ is therefore equivalent to estimating $\theta_{0}$ given independent observations $\mathbf{O}_{1}, \ldots, \mathbf{O}_{n}$ from $P_{0}$, where $n_{1}:=\sum_{i=1}^{n} D_{i}$.

The benefit to the above reframing of the problem is that $\theta_{0}, F_{0}$, and $G_{0}$ can then be written as transformations of $P_{0}$. First, we have that $\theta_{0}(z)=$ $T\left(\mu_{0}(z)\right) / T\left(\pi_{0}\right)$, where $\mu_{0}(z):=P_{0}(D=1 \mid Z=z)$ and $T:[0,1) \rightarrow \mathbb{R}^{+}$is 
the odds transformation, defined as $T(\mu):=\mu /(1-\mu)$. Since $T$ is strictly increasing, $\theta_{0}$ is monotone if and only if $\mu_{0}$ is. Since the maximum likelihood estimator of $\mu_{0}$ under the assumption that $\mu_{0}$ is non-decreasing is given by the isotonic regression $\mu_{n}^{*}$ of $D_{1}, \ldots, D_{n}$ on $Z_{1}, \ldots, Z_{n}$, and the maximum likelihood estimator of $\pi_{0}$ is given by $\pi_{n}$, the maximum likelihood estimator of $\theta_{0}(z)$ is then given by $T\left(\mu_{n}^{*}(z)\right) / T\left(\pi_{n}\right)$. It is straightforward to see that this form of the maximum likelihood estimator is equivalent to the forms given above. In the next section, we will utilize this form of $\theta_{n}^{*}$ to derive its asymptotic properties and to construct asymptotic confidence intervals.

\section{Asymptotic results}

\subsection{Discrete distributions}

We first consider the situation where $G_{0}$ has finite support $\mathcal{G}_{0}$ and $\theta_{0}$ is strictly increasing on $\mathcal{G}_{0}$. The next result demonstrates that in this case, $F_{n}^{*}$ and $G_{n}^{*}$ are asymptotically equivalent to $F_{n}$ and $G_{n}$, respectively, and $\theta_{n}^{*}$ is asymptotically equivalent to the ratio of the empirical masses on the support of $G_{0}$.

Theorem 3 (Discrete distributions). Suppose that the support $\mathcal{G}$ of $G_{0}$ is a finite set $\left\{y_{1}<y_{2}<\cdots<y_{m_{2}}\right\}$ and that $\left[F_{0}\left(y_{j}\right)-F_{0}\left(y_{j-1}\right)\right] / \Delta G_{0}\left(y_{j}\right)<$ $\left[F_{0}\left(y_{j+1}\right)-F_{0}\left(y_{j}\right)\right] / \Delta G_{0}\left(y_{j+1}\right)$ for $j=1, \ldots, m_{2}-1$, where $y_{0}:=-\infty$. 
4.2 Continuous distributions16

Then $F_{n}^{*}=F_{n}$ and $G_{n}^{*}=G_{n}$ with probability tending to one, so that with probability tending to one $\theta_{n}^{*}$ is a left-continuous step function with jumps at $y_{1}, \ldots, y_{m_{2}-1}$ and $\theta_{n}^{*}\left(y_{j}\right)=\left[F_{n}\left(y_{j}\right)-F_{n}\left(y_{j-1}\right)\right] / \Delta G_{n}\left(y_{j}\right)$ and $\theta_{n}^{*}(z)=0$ for $z<y_{1}$. As a result, $n^{1 / 2}\left[\theta_{n}^{*}\left(y_{j}\right)-\theta_{0}\left(y_{j}\right)\right] \stackrel{\mathrm{d}}{\longrightarrow} N\left(0, \sigma_{0}^{2}\left(y_{j}\right)\right)$ for

$$
\sigma_{0}^{2}\left(y_{j}\right):=\theta_{0}\left(y_{j}\right) \frac{\pi_{0} F_{0, j}+\left(1-\pi_{0}\right) \Delta G_{0}\left(y_{j}\right)-F_{0, j} \Delta G_{0}\left(y_{j}\right)}{\pi_{0}\left(1-\pi_{0}\right)\left[\Delta G_{0}\left(y_{j}\right)\right]^{2}}
$$

where $F_{0, j}:=F_{0}\left(y_{j}\right)-F_{0}\left(y_{j-1}\right)$.

We note that the above result does not require that $F_{0}$ be discrete as well, or be dominated by $G_{0}$. If $F_{0}$ is dominated by $G_{0}$, then $\theta_{0}=\Delta F_{0} / \Delta G_{0}$ corresponds to the ratio of the mass functions.

\subsection{Continuous distributions}

Now we address the situation where $F_{0}$ and $G_{0}$ are both absolutely continuous on $\mathcal{G}_{0}$ and $\theta_{0}$, which now corresponds to the ratio $f_{0} / g_{0}$ of the density functions, is strictly increasing. We first consider the large-sample behavior of $F_{n}^{*}$ and $G_{n}^{*}$.

Theorem 4. Suppose that $G_{0}$ is supported on a bounded interval $[a, b] \subset \mathbb{R}$, that $F_{0}$ and $G_{0}$ possess continuous density functions $f_{0}$ and $g_{0}$ on $[a, b]$ such that $f_{0} / g_{0}$ is strictly increasing on $[a, b]$, and $g_{0}(z) \geq \kappa>0$ on $[a, b]$. Then $\left\|G_{n}^{*}-G_{n}\right\|_{\infty}=o_{P}\left(n^{-1 / 2}\right)$ and $\left\|F_{n}^{*}-F_{n}\right\|_{\infty}=o_{P}\left(n^{-1 / 2}\right)$. 
4.2 Continuous distributions17

Theorem 4 demonstrates that when $\theta_{0}$ is strictly increasing, the maximum likelihood estimators of the individual distribution functions are asymptotically equivalent to the empirical distribution functions at the rate $n^{-1 / 2}$, and hence possess the same limit distributions as the empirical distribution functions. This result is proved using the functional delta method and the results of Beare and Fang (2017), who demonstrated that the LCM operation is a directionally Hadamard differentiable mapping at any concave function.

We now turn to large-sample results for $\theta_{n}^{*}$ at points $z$ where both $F_{0}$ and $G_{0}$ possess Lebesgue density functions $f_{0}$ and $g_{0}$, respectively. First, consistency of $\mu_{n}^{*}$ implies consistency of $\theta_{n}^{*}$.

Theorem 5 (Consistency). If $f_{0}$ is continuous at $x, g_{0}$ is continuous at $x$, and $g_{0}(x)>0$, then $\theta_{n}^{*}(x) \stackrel{\mathrm{P}}{\longrightarrow} \theta_{0}(x)$. If $f_{0}$ and $g_{0}$ are uniformly continuous on $\mathcal{G}_{0}$, then $\sup _{x \in I}\left|\theta_{n}^{*}(x)-\theta_{0}(x)\right| \stackrel{\mathrm{P}}{\longrightarrow} 0$ for any strict sub-interval $I \subsetneq \mathcal{G}_{0}$.

We recall that, at any $z$ such that $h_{0}=\pi_{0} f_{0}+\left(1-\pi_{0}\right) g_{0}$ is positive and continuous in a neighborhood of $z, \mu_{0}(z) \in(0,1)$, and $\mu_{0}$ is continuously differentiable in a neighborhood of $z$, it holds that

$$
n^{1 / 3}\left[\mu_{n}^{*}(z)-\mu_{0}(z)\right] \stackrel{\mathrm{d}}{\longrightarrow}\left\{4 \mu_{0}^{\prime}(z) \mu_{0}(z)\left[1-\mu_{0}(z)\right] h_{0}(z)^{-1}\right\}^{1 / 3} W
$$

where $W$ follows Chernoff's distribution, defined as the point of maximum 
4.2 Continuous distributions18

of $Z(u)-u^{2}$ for $Z$ a two-sided standard Brownian motion originating from zero (Brunk, 1970; Groeneboom and Jongbloed, 2014). We can then use the delta-method to see that

$n^{1 / 3}\left[\theta_{n}^{*}(z)-\theta_{0}(z)\right] \stackrel{\mathrm{d}}{\longrightarrow} T\left(\pi_{0}\right) T^{\prime}\left(\mu_{0}\right)\left\{4 \mu_{0}^{\prime}(z) \mu_{0}(z)\left[1-\mu_{0}(z)\right] h_{0}(z)^{-1}\right\}^{1 / 3} W$

The scale parameter in the above limit distribution is equal to $\left[4 \kappa_{0}(z) \theta_{0}^{\prime}(z)\right]^{1 / 3}$

for

$$
\kappa_{0}(z):=\theta_{0}(z) \frac{\pi_{0} f_{0}(z)+\left(1-\pi_{0}\right) g_{0}(z)}{\pi_{0}\left(1-\pi_{0}\right) g_{0}(z)^{2}}
$$

This yields the following result.

Theorem 6 (Pointwise convergence in distribution). Suppose that, in a neighborhood of $z, \theta_{0}$ is continuously differentiable with $\theta_{0}^{\prime}(z)>0$, and $f_{0}$ and $g_{0}$ are positive and continuous. Then $n^{1 / 3}\left[\theta_{n}^{*}(z)-\theta_{0}(z)\right] \stackrel{\mathrm{d}}{\longrightarrow}\left[4 \kappa_{0}(z) \theta_{0}^{\prime}(z)\right]^{1 / 3} W$.

Theorem 6 reflects certain common tradeoffs in the monotonicity-constrained literature. Theorem 6 indicates that the non-smoothed estimator converges pointwise at the $n^{-1 / 3}$ rate. In contrast, the smoothed estimator proposed by $\mathrm{Yu}$ et al. (2017) converges at the faster $n^{-2 / 5}$ rate, albeit under stronger smoothness assumptions. While $\mathrm{Yu}$ et al. (2017) did not propose a method for conducting inference, smoothed estimators typically possess an asymptotic bias that complicates the task of performing valid inference. In contrast, the limit distribution in Theorem 6 has mean zero, which we 
4.2 Continuous distributions19

can use to construct asymptotically valid confidence intervals. Defining $\tau_{n}(z)$ as an estimator of $\tau_{0}(z):=\kappa_{0}(z) \theta_{0}^{\prime}(z)$ and $q_{\alpha}$ the $1-\alpha / 2$ quantile of $W$, a $100(1-\alpha) \%$ Wald-type confidence interval for $\theta_{0}(z)$ is given by $\theta_{n}^{*}(z) \pm\left[4 \tau_{n}(z) / n\right]^{1 / 3} q_{1-\alpha / 2}$. If $\tau_{n}(z) \stackrel{\mathrm{P}}{\longrightarrow} \tau_{0}(z)$, then this interval has asymptotic coverage of $100(1-\alpha) \%$. The quantiles of $W$ were computed by Groeneboom and Wellner $(2001)$, and in particular $q_{0.975} \approx 0.9982$.

In practice, we recommend an alternative method to constructing confidence intervals for $\theta_{0}(z)$. We recommend first constructing confidence intervals for $\mu_{0}(z)$ using either of two existing methods, then transforming these intervals into intervals for $\theta_{0}(z)$. Specifically, if $\left[\ell_{n}(z), u_{n}(z)\right]$ represents a $100(1-\alpha) \%$ confidence interval for $\mu_{0}(z)$, then we take $\left[T\left(\ell_{n}(z)\right) / T\left(\pi_{n}\right)\right.$, $\left.T\left(u_{n}(z)\right) / T\left(\pi_{n}\right)\right]$ as a $100(1-\alpha) \%$ confidence interval for $\theta_{0}(z)$. Two existing ways to construct $\left[\ell_{n}(z), u_{n}(z)\right]$ are Wald-type intervals with plug-in estimation of nuisance parameters and intervals based on likelihood ratio tests. The former intervals are analogous to the Wald-type interval, but based on the limit distribution for $n^{1 / 3}\left[\mu_{n}^{*}(z)-\mu_{0}(z)\right]$ given in $(4.2)$. Alternatively, confidence intervals obtained by inverting likelihood ratio tests, proposed first by Banerjee and Wellner (2001) and studied further by, e.g. Banerjee (2007) and Groeneboom and Jongbloed (2015), can be formed based on the limiting distribution of twice the log of the ratio of the likelihoods of 
the maximum likelihood estimator and a suitably constrained maximum likelihood estimator. Since this limiting distribution is pivotal, meaning it does not depend on any unknown features of the true distribution, this approach does not require estimating any unknown nuisance parameters. We therefore expect this method to have better finite-sample properties than intervals based on plug-in estimation of nuisance parameters.

\section{Numerical studies}

In Supplementary Material, we present results of two simulation studies in the cases where $F_{0}$ and $G_{0}$ are fully discrete and fully continuous. In short, these studies confirm the validity of our large-sample theory and demonstrate that the maximum likelihood estimator and various proposed methods of conducting inference perform well in both cases. Here, we present the results of a numerical study illustrating the behavior of $\theta_{n}^{*}$ when $F_{0}$ and $G_{0}$ are mixed discrete-continuous distributions. We note that our asymptotic results did not address the behavior of $\theta_{n}^{*}$ at mass points in mixed discretecontinuous distributions; to the best of our knowledge, no such results yet exist for monotone estimators. We use this numerical study to explore this important case.

We simulated $Y$ as a mixed discrete-continuous random variable with 
probability $1 / 9$ each of being $0,0.5$ and 1 , and probability $2 / 3$ of being from the uniform distribution on $[0,1]$, and simulated $X$ as a mixed discretecontinuous random variable with probabilities $1 / 18,1 / 9$, and $3 / 18$ of being $0,0.5$, and 1 , respectively and probability $2 / 3$ of being from the density function $x \mapsto I_{[0,1]}(x)(0.5+x)$. We then have that $\theta_{0}(x)=0.5+x$ for $x \in[0,1]$ We set $\pi_{0}:=0.4$. For each combined sample size $n \in\{500,1 K, 5 K, 10 K\}$, we simulated 1000 datasets, and in each dataset we computed the maximum likelihood estimator, the maximum smoothed likelihood estimator of Yu et al. (2017), and the non-monotone estimator based on kernel density estimates for each $z \in\{0,0.05, \ldots, 0.95,1\}$. We constructed confidence intervals at each $z$ using the transformed plug-in and likelihood ratio-based methods described in Section 4.2. To estimate the scale parameter in the limit distribution of $\mu_{n}^{*}(z)$ as defined in equation 4.2, we used the plug-in estimator $\mu_{n}^{*}(z)$ for $\mu_{0}(z)$ and estimated $\mu_{0}^{\prime}(z) / h_{0}(z)=\left(\mu_{0} \circ H_{0}^{-1}\right)^{\prime} \circ H_{0}(z)$ using the derivative of a local linear smoother of $\mu_{n}^{*} \circ H_{n}^{-}$evaluated at $H_{n}(z)$. In addition to the properties of the estimators listed above, we also investigated the properties of the general sample-splitting procedure proposed by Banerjee et al. (2019). Given a generic monotone estimator $\gamma_{n}$ of a monotone function $\gamma_{0}$ such that $n^{1 / 3}\left[\gamma_{n}(z)-\gamma_{0}(z)\right] \stackrel{\mathrm{d}}{\longrightarrow} G$ for $G$ a meanzero distribution with finite variance, Banerjee et al. (2019) proposed ran- 
domly splitting the sample into $m$ subsets of roughly equal size, computing monotone estimates $\gamma_{n, 1}, \ldots, \gamma_{n, m}$ in each subset, then defining $\bar{\gamma}_{n, m}(z):=$ $\frac{1}{m} \sum_{j=1}^{m} \gamma_{n, j}(z)$. They demonstrated that if $m>1$ is fixed, then under mild conditions $\bar{\gamma}_{n, m}(z)$ has strictly better asymptotic mean squared error than $\gamma_{n}(z)$, and that for moderate $m, \bar{\gamma}_{n, m}(z) \pm \sigma_{n, m}(z) t_{1-\alpha / 2, m-1} / \sqrt{m}$ forms an asymptotic $100(1-\alpha) \%$ confidence interval for $\gamma_{0}(z)$, where $\sigma_{n, m}^{2}(z):=$ $\frac{1}{m-1} \sum_{j=1}^{m}\left[\gamma_{n, j}(z)-\bar{\gamma}_{n, m}(z)\right]^{2}$ and $t_{1-\alpha / 2, m-1}$ is the $100(1-\alpha / 2)$ quantile of the $t$-distribution with $m-1$ degrees of freedom. Therefore, $\bar{\gamma}_{n, m}(z)$ is preferable to $\gamma_{n}(z)$ for two reasons: it has better asymptotic mean squared error, and asymptotically valid pointwise confidence intervals for $\gamma_{0}$ based on $\bar{\gamma}_{n, m}$ can be formed without estimating any nuisance parameters. They also studied the asymptotic properties of $\bar{\gamma}_{n, m_{n}}(z)$ when $m_{n}$ grows with $n$. In our simulation study, we considered the estimator $\bar{\theta}_{n, m}$ defined as $\bar{\theta}_{n, m}(z):=\frac{1}{m} \sum_{j=1}^{m} \theta_{n, j}^{*}(z)$, where $\theta_{n, j}^{*}$ is the maximum likelihood estimator in the $j$ th subset, and the corresponding confidence intervals defined above. We only considered the situation where $m \in\{5,10\}$ is fixed with the sample size.

We now turn to the results of the simulation study. The left panel of Figure 1 displays the distribution of $\theta_{n}^{*}(z)-\theta_{0}(z)$ for $z \in[0,1]$ and $n=10 K$. These distributions are approximately centered around 0 for $z \in(0,1)$, but 

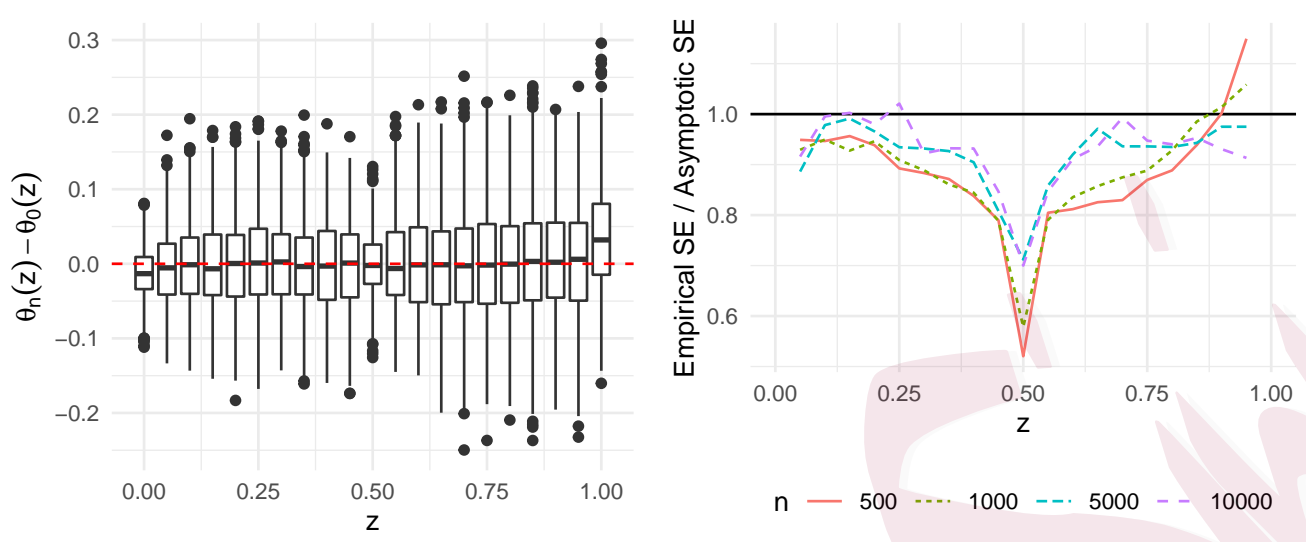

Figure 1: Left: boxplots of $\theta_{n}^{*}(z)-\theta_{0}(z)$ with $n=10 K$. Right: empirical standard errors of $r_{n}\left[\theta_{n}^{*}(z)-\theta_{0}(z)\right]$ divided by the limit theory-based counterparts for $z \in(0,1)$, where $r_{n}=n^{1 / 2}$ for $z=0.5$ and $r_{n}=n^{1 / 3}$ otherwise.

not for $z \in\{0,1\}$. Hence, despite the positive mass at the boundaries, the maximum likelihood estimator does not appear to be consistent at the boundaries. This is a common problem among monotonicity-constrained estimators, and various correction procedures have been proposed and could be considered in this context (see, e.g. Woodroofe and Sun, 1993; Kulikov and Lopuhaä, 2006).

The right panel of Figure 1 displays the ratio of the standard deviation of $r_{n}\left[\theta_{n}^{*}(z)-\theta_{0}(z)\right]$ to the standard deviation of the asymptotic distributions derived in Section 4 for $z \neq 0,1$. For $z=0.5, r_{n}=n^{1 / 2}$ and the asymptotic 
distribution is that of the fully discrete case presented in Section 4.1 , though we note that the results presented in that section do not apply here due to the mixed discrete-continuous nature of $F_{0}$ and $G_{0}$ here. Otherwise, $r_{n}=$ $n^{1 / 3}$ and the asymptotic distribution is that of the continuous case presented in Section 4.2. We see that, for $z \neq 0.5$, the empirical standard error approaches the asymptotic standard deviation as $n$ grows. However, for $z=0.5$, the empirical standard error is converging to a limit that is strictly smaller than the asymptotic standard deviation. This suggests that, at points that have both positive mass and positive density in a neighborhood of the point, the maximum likelihood estimator gains efficiency from the positive density. In addition, points of continuity near the mass point also experience finite-sample efficiency gains.

Figure 2 shows the ratio of the mean squared errors of the maximum smoothed likelihood estimator, the kernel density-based estimator, and the sample splitting estimators to that of the maximum likelihood estimator. The maximum smoothed likelihood estimator is slightly more efficient than the maximum likelihood estimator at continuity points, but is less efficient around mass points. Furthermore, the relative performance of the maximum likelihood estimator at positive mass points increases as the sample size grows. The kernel density estimator is generally less efficient than 
the maximum likelihood estimator, especially near mass points, and the discrepancy also grows with the sample size.

For large enough $n$, the sample splitting estimator is more efficient than the maximum likelihood estimator at all points at which the latter is consistent. The relative improvement of $\bar{\theta}_{n, m}$ grows with the number of splits $m$, as does the sample size $n$ required for $\bar{\theta}_{n, m}$ to outperform $\theta_{n}^{*}$.

Figure 3 shows the empirical coverage of $95 \%$ confidence intervals for $\theta_{0}(z)$ constructed using the plug-in method described in Section 4.2, the inverted likelihood ratio test approach of Banerjee and Wellner $(2001)$, and the sample splitting approach of Banerjee et al. (2019) described above. We note that the likelihood ratio approach does not provide intervals at the end points $z=0$ or $z=1$. The plug-in method is conservative in large samples near mass points, but anti-conservative at some points of positive density. This is because the plug-in method is designed to work when the distributions are fully continuous, and estimation of the required nuisance parameters in the limit distribution fails in the presence of mass points. The likelihood ratio method is conservative in smaller samples, but approaches nominal coverage in large samples for points $z$ of absolute continuity. The sample splitting method with $m=5$ has adequate coverage for all sample sizes except for $z$ close to the boundaries. The sample splitting method with 


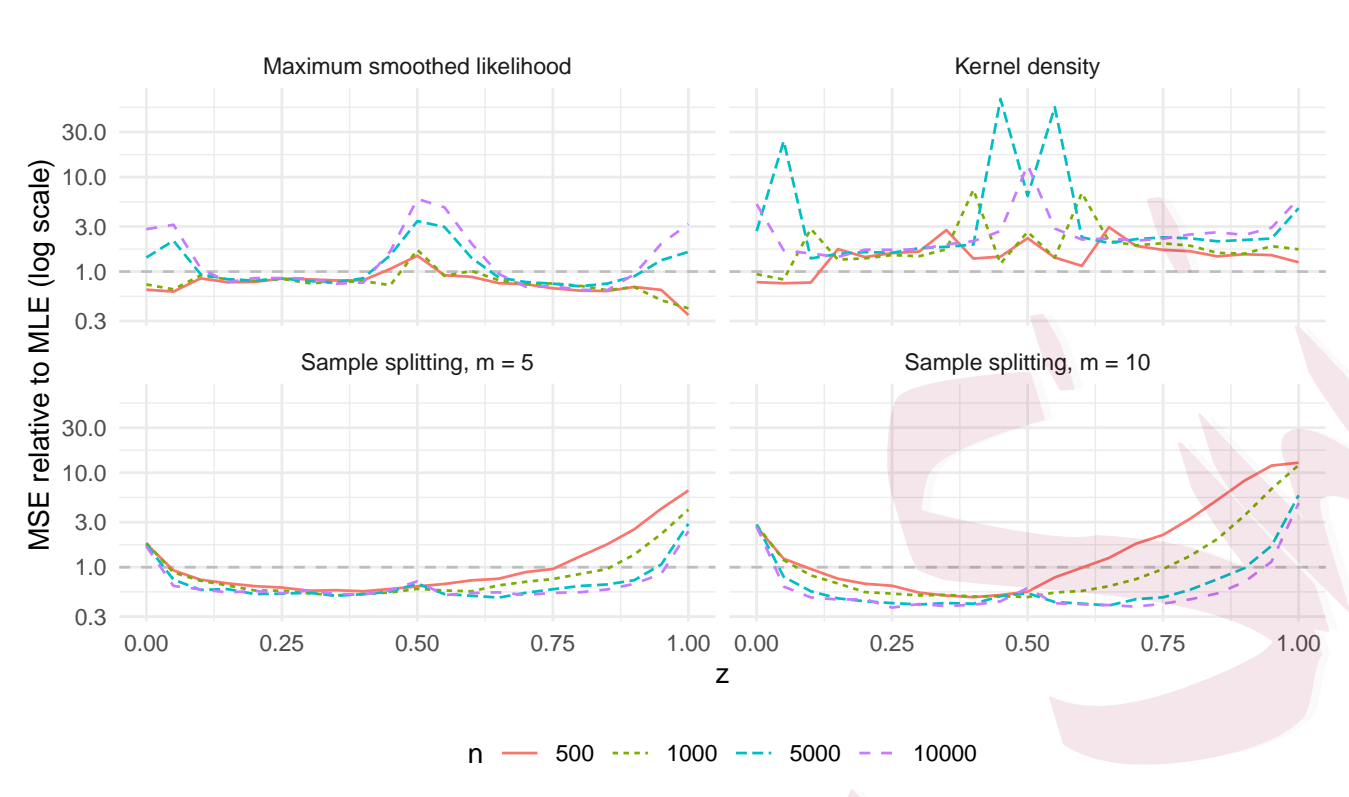

Figure 2: Relative mean squared errors of the maximum smoothed likelihood estimator, the kernel density-based estimator, and the sample splitting estimators to the maximum likelihood estimator for $z \in[0,1]$ and various sample sizes $n$. The maximum likelihood has better mean squared error for $y$-values greater than one, and the other estimator has better mean squared error for $y$-values less than one.

$m=10$ (and similarly for $m=20$, which is not shown) appears to require very large sample sizes to attain adequate coverage over a large range of z. We note that the sample splitting methods was able to achieve good coverage in large samples at both interior absolutely continuous points and interior mass points, without the user specifying which points are which. 


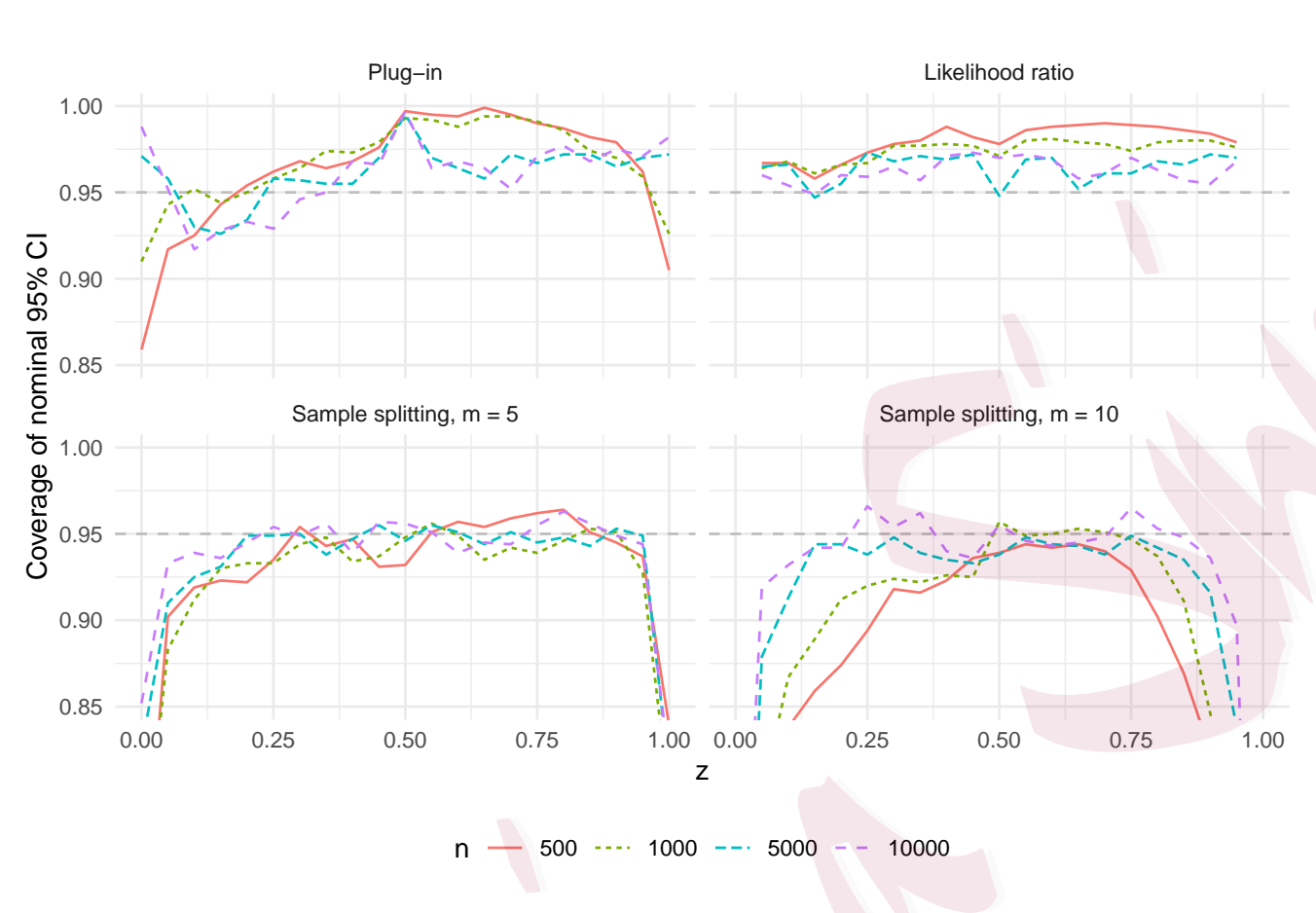

Figure 3: Coverage of $95 \%$ CIs for $z \in[0,1]$, various sample sizes $n$, and four methods: the plug-in method centered around the maximum likelihood estimator (upper left), the inverted likelihood ratio tests (upper right), and the sample splitting method with $m=5$ (lower left) and $m=10$ (lower right). Note that the likelihood ratio method does not provide intervals at the endpoints. 


\section{Analysis of C-reactive protein for predicting bacterial infec- tion}

In this section, we use the methods presented herein to assess the use of the biomarker C-reactive protein (CRP) for determining the presence or absence of bacterial infection in children with systemic inflammatory response syndrome (SIRS). The Optimizing Antibiotic Strategies in Sepsis (OASIS) II study enrolled a prospective observational cohort of children under the age of nineteen at the pediatric intensive care unit at The Children's Hospital of Philadelphia from August 2012 to June 2016 (Downes et al., 2018). Patients were enrolled in the study if they presented signs of SIRS, were started on a new broad-spectrum antibiotic for suspected bacterial infection, and had blood cultures taken within six hours of SIRS onset. A primary goal of the study was to assess whether CRP, which had previously been found to be predictive of bacterial infection (Downes et al. 2017), could be used to determine when antibiotic therapy could be safely ended. Additional details of the study design and results of the primary analysis may be found in Downes et al. (2018).

We analyzed all patients in the OASIS II cohort with measured biomarkers and bacterial infection status to assess the odds of bacterial infection as a function of CRP value. Some patients had measurements at multiple 
episodes; since all such episodes were at least 30 days apart, we treated these episodes as independent of one another. We analyzed a total of $n=504$ CRP measurements among 443 unique patients, with $n_{1}=202$ bacterial infections among 191 unique patients and $n_{2}=302$ non-infections among 266 unique patients.

Since CRP has previously been found to be predictive of bacterial infection in this patient population, there is scientific reason to believe that the density ratio order holds. We therefore computed the MLE of the density ratio function and corresponding $95 \%$ likelihood ratio-based pointwise confidence intervals and the sample splitting estimator of Banerjee et al. (2019) with $m=5$ splits and corresponding $95 \%$ pointwise confidence intervals.

Figure 4 displays the estimated odds of bacterial infection given CRP value relative to the population odds of bacterial infection and $95 \%$ pointwise confidence intervals. We find that values of CRP under 1 are indicative of roughly quartered odds of infection relative to the population odds of infection, and values of CRP greater than 20 are indicative of roughly doubled odds of infection relative to the population odds. Values of CRP between 1 and 20 do not clearly indicate that a patient's odds of infection are larger or smaller than the population odds. 


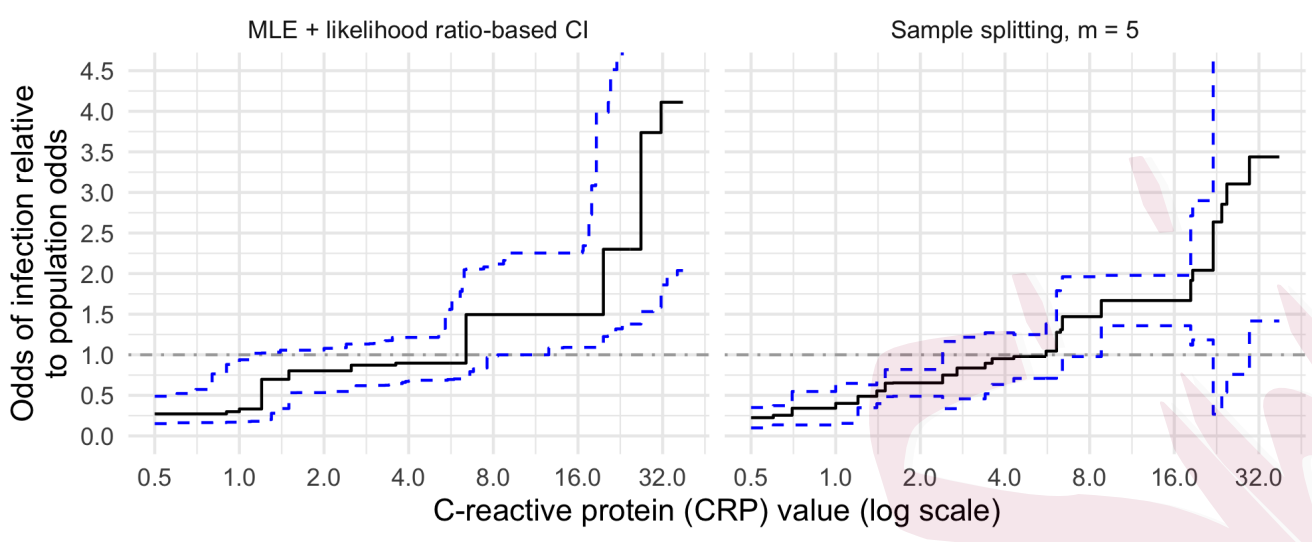

Figure 4: Odds of bacterial infection given C-reactive protein value relative to population odds in children with treating systemic inflammatory response syndrome.

\section{Discussion}

In this article, we have considered nonparametric maximum likelihood inference for the density ratio function and the individual distribution functions under the assumption that the density ratio is nondecreasing. We applied these methods to the analysis of the biomarker C-reactive protein for predicting bacterial infection in children with systemic inflammatory response syndrome. The methods apply broadly to biomarker analysis, as well as other areas of biomedical research.

One of our important contributions is the ability to deal with discrete, continuous, and mixed discrete-continuous distributions. Such distribu- 
tions arise frequently in applied settings, and in particular in the context of biomarker analysis. Furthermore, we have demonstrated via numerical studies that sample splitting provides good pointwise confidence interval coverage without knowing which values correspond to discrete mass points and which correspond to points of Lebesgue continuity of the underlying densities, which is important because in practice analysts may not have such knowledge a priori. However, a theoretical treatment of the precise asymptotic behavior of the estimator at mass points remains unknown, and would be an interesting topic of future research.

\section{Supplementary Materials}

The Supplementary Materials contain an example of the use of Theorem 2, proofs of all theorems, additional simulation results, and additional results from the data analysis.

\section{Acknowledgements}

The authors thank Craig Boge for help compiling the OASIS II data. The authors also gratefully acknowledge the constructive comments of the editors and anonymous reviewers and the support of the CDC Epicenters program (KJD), NICHD grant K23HD091365 (KJD), the Center for Pediatric Clinical Effectiveness and the Pediatric IDEAS Research Group of 
the Children's Hospital of Philadelphia (KJD, TW), and the Department of Pediatrics of the University of Pennsylvania Perelman School of Medicine.

\section{References}

Arcones, M. A. and F. J. Samaniego (2000). On the Asymptotic Distribution Theory of a Class of Consistent Estimators of a Distribution Satisfying a Uniform Stochastic Ordering Constraint. Ann. Stat. 28(1), 116-150.

Bamber, D. (1975). The area above the ordinal dominance graph and the area below the receiver operating characteristic graph. J. Math. Psychol. 12(4), $387-415$.

Banerjee, M. (2007, 07). Likelihood based inference for monotone response models. Ann. Statist. 35(3), 931-956.

Banerjee, M., C. Durot, and B. Sen (2019, 04). Divide and conquer in nonstandard problems and the super-efficiency phenomenon. Ann. Statist. 47(2), 720-757.

Banerjee, M. and J. A. Wellner (2001). Likelihood ratio tests for monotone functions. Ann. Statist. 29(6), 1699-1731.

Beare, B. K. and Z. Fang (2017). Weak convergence of the least concave majorant of estimators for a concave distribution function. Electron. J. Statist. 11(2), 3841-3870.

Beare, B. K. and J.-M. Moon (2015). Nonparametric tests of density ratio ordering. Econom. Theory 31(3), 471-492.

Brunk, H. D. (1970). Estimation of isotonic regression. In Nonparametric Techniques in Sta- 


\section{REFERENCES33}

tistical Inference (Proc. Sympos., Indiana Univ., Bloomington, Ind., 1969), London, pp. 177-197. Cambridge Univ. Press.

Carolan, C. A. and J. M. Tebbs (2005, 03). Nonparametric tests for and against likelihood ratio ordering in the two-sample problem. Biometrika 92(1), 159-171.

Davidov, O. and A. Herman (2012). Ordinal dominance curve based inference for stochastically ordered distributions. J. R. Stat. Soc. Ser. B. Stat. Methodol. 74(5), 825-847.

Downes, K. J., J. C. Fitzgerald, E. Schriver, et al. (2018). Implementation of a Pragmatic Biomarker-Driven Algorithm to Guide Antibiotic Use in the Pediatric Intensive Care Unit: the Optimizing Antibiotic Strategies in Sepsis (OASIS) II Study. J. Pediatric Infect. Dis. Soc.. advance online publication.

Downes, K. J., S. L. Weiss, J. S. Gerber, et al. (2017). A Pragmatic Biomarker-Driven Algorithm to Guide Antibiotic Use in the Pediatric Intensive Care Unit: The Optimizing Antibiotic Strategies in Sepsis (OASIS) Study. J. Pediatric Infect. Dis. Soc. 6(2), 134-141.

Dykstra, R., S. Kochar, and T. Robertson (1995). Inference for likelihood ratio ordering in the two-sample problem. J. Amer. Statist. Assoc. 90(431), 1034-1040.

Groeneboom, P. and G. Jongbloed (2014). Nonparametric estimation under shape constraints. Cambridge University Press.

Groeneboom, P. and G. Jongbloed $(2015,10)$. Nonparametric confidence intervals for monotone functions. Ann. Statist. 43(5), 2019-2054. 


\section{REFERENCES34}

Groeneboom, P. and J. A. Wellner (2001). Computing Chernoff's distribution. J. Comput. Graph. Statist. 10(2), 388-400.

Hsieh, F. and B. W. Turnbull (1996, 02). Nonparametric and semiparametric estimation of the receiver operating characteristic curve. Ann. Statist. 24(1), 25-40.

Kulikov, V. N. and H. P. Lopuhaä (2006, 04). The behavior of the NPMLE of a decreasing density near the boundaries of the support. Ann. Statist. 34(2), 742-768.

Lehmann, E. L. and J. Rojo (1992, 12). Invariant Directional Orderings. Ann. Statist. 20(4), 2100-2110.

Mukerjee, H. (1996). Estimation of survival functions under uniform stochastic ordering. $J$. Amer. Statist. Assoc. 91(436), 1684-1689.

Rojo, J. and F. J. Samaniego (1991). On nonparametric maximum likelihood estimation of a distribution uniformly stochastically smaller than a standard. Statist. Probab. Lett. 11(3), $267-271$.

Rojo, J. and F. J. Samaniego (1993). On estimating a survival curve subject to a uniform stochastic ordering constraint. J. Amer. Statist. Assoc. 88(422), 566-572.

Roosen, J. and D. A. Hennessy (2004). Testing for the monotone likelihood ratio assumption. J. Bus. Econ. Stat. 22(3), 358-366.

Shaked, M. and J. G. Shanthikumar (2007). Stochastic Orders. Springer Science \& Business Media. 


\section{REFERENCES35}

Tang, C.-F., D. Wang, and J. M. Tebbs (2017, 12). Nonparametric goodness-of-fit tests for uniform stochastic ordering. Ann. Statist. 45(6), 2565-2589.

Woodroofe, M. and J. Sun (1993). A penalized maximum likelihood estimate of $f(0+)$ when $f$ is non-increasing. Statist. Sinica 3(2), 501-515.

Yu, T., P. Li, and J. Qin (2017). Density estimation in the two-sample problem with likelihood ratio ordering. Biometrika $104(1), 141-152$.

Department of Mathematics and Statistics, University of Massachusetts Amherst

E-mail: twestling@math.umass.edu

Center for Pediatric Clinical Effectiveness, Children's Hospital of Philadelphia

E-mail: downeskj@email.chop.edu

Department of Statistics, The Wharton School, University of Pennsylvania

E-mail: dsmall@wharton.upenn.edu 